\title{
Strong Field Molecular Ionization to Multiple Ionic States: Direct vs Indirect Pathways
}

\author{
Péter Sándor ${ }^{1}$, Arthur Zhao ${ }^{1}$, Tamás Rozgonyi ${ }^{2}$, Thomas \\ Weinacht $^{1}$ \\ ${ }^{1}$ Department of Physics and Astronomy, Stony Brook University, 100 Nicolls Road, \\ Stony Brook, NY 11794-3800, USA \\ ${ }^{2}$ Institute of Materials and Environmental Chemistry, Research Centre for Natural \\ Sciences, Hungarian Academy of Sciences, Budapest 1025, Pusztaszeri út 59-67, \\ Hungary
}

\begin{abstract}
We present velocity map imaging measurements of photoelectrons in coincidence with ions produced via strong field molecular ionization. Our measurements, in conjunction with electronic structure and Stark shift calculations, allow us to assign several features in the low energy portion of the photoelectron spectrum to different molecular electronic continua (ionic states). Furthermore, we are able to distinguish between direct and indirect ionization pathways, uncovering the role of both neutral and ionic resonances in the ionization dynamics.
\end{abstract}




\section{Introduction}

Strong Field Ionization plays a key role in high harmonic generation and attosecond electron dynamics (Kling \& Vrakking 2008, Agostini \& DiMauro 2004). It has also been used as a probe of excited state molecular dynamics (Li et al. 2010). While early works suggested that many experiments could be interpreted in terms of simply removing the most weakly bound electron (i.e. from the Highest Occupied Molecular Orbital - HOMO), recent experiments (as well as one earlier work - (Gibson et al. 1991)) have highlighted the role of ionization from multiple orbitals (Li et al. 2008, McFarland et al. 2008, Spanner et al. 2012, Kotur et al. 2012). Understanding what states of the molecular cation are populated, and how, is very important for producing and probing electron wave packets, as well as for understanding strong field ionization as a tool for molecular imaging and following neutral dynamics. Here we build upon a recent work which established direct ionization to multiple states of the molecular cation via coincidence detection of electrons and ions (Boguslavskiy et al. 2012). We make use of coincidence detection of photoelectrons and ions with velocity map imaging of the photoelectrons (Lehmann et al. 2012). Furthermore, by combining our coincidence measurements with $a b$ initio electronic structure and dynamic Stark shift calculations for the molecular cations, we are able to assign peaks in the photoelectron spectrum to specific ionic states, and determine the amount of direct vs indirect ionization to each state.

\section{Experiment}

Our experimental apparatus consists of an amplified Ti:sapphire laser system, which produces pulses with $1 \mathrm{~mJ}$ pulse energy, 30 fs transform limited pulse duration (intensity FWHM), a central wavelength of $780 \mathrm{~nm}$ and $1 \mathrm{kHz}$ repetition rate. The linearly polarized laser beam crosses the effusive molecular beam in a vacuum chamber. Here we generate charged particles, which are detected by accelerating them toward a dual stack of microchannel plates and phosphor screen with an electrostatic lens configured for velocity map imaging (VMI) of the charged particles, producing a two dimensional projection of the three dimensional charged particle velocity distribution (Eppink \& Parker 1997). The hit locations on the phosphor screen are recorded and digitized by a CMOS camera. Experiments can be run in either "regular" VMI or coincidence VMI mode.

During regular velocity map imaging data acquisition, constant $-950,-670$ and $0 \mathrm{~V}$ are applied to the back, middle and front plates of the electrostatic lenses, respectively, collecting the electrons only. The number of electrons generated per laser shot is much larger than one, with the exact number depending on the experiment in question. The camera integration time is much longer than the laser repetition period (1 ms). This way, the raw images already contain $\approx 10^{5}$ electron hits, which results in a $2 \mathrm{D}$ photoelectron momentum distribution with a signal to noise ratio allowing for Abel inversion of the 
image.

In coincidence velocity map imaging mode, the goal is to measure an electron and ion from the same molecule. Thus for $100 \%$ detection efficiency, one would aim to maximize the probability of ionizing one molecule per laser shot and simply discard laser shots that produced two or more ions. However, with less than $100 \%$ detection efficiency, one has to worry about false coincidences - ions and electrons that come from separate molecules and whose partner electron or ion is not detected. Based upon a detailed analysis of the true and false coincidence rates for our detection efficiencies (see appendix I), we set the experimental parameters (laser intensity and molecular beam density) such that the number of molecules ionized per laser shot to be slightly below one - it was set to be about 0.5 for the coincidence measurements discussed in this paper. The repeller plate voltages are switched from -600 to $+1000 \mathrm{~V}$ on the back plate, and from -420 to $+700 \mathrm{~V}$ on the middle plate whithin $\approx 300 \mathrm{~ns}(10 \%$ to $90 \%$ voltage level). (The front plate stays grounded.) The switching starts immediately after an electron is detected on the MCP. After switching is done, the positively charged ion is collected. The ion collection efficiency of course strongly depends on the timing of the switching, while its detection efficiency depends on the accelerating voltage. The latter was limited by the range our high voltage switch could support (a total of $1.6 \mathrm{kV}$ ). The integration time of the camera is much smaller than the laser repetition period (usually $24 \mu \mathrm{s}$ ), and synchronized with the laser pulse in such a way that once the location of the electron hit is recorded, the shutter closes. The ionic fragment, which hits the MCP microseconds later, is identified based on its time of flight. A computer algorithm calculates the coordinates of the electron hit location, and pairs it with the label of the ionic fragment. During post-processing, a 2D photoelectron momentum distribution is synthetized for each fragment from the coordinates for each electron hit. Data acquisition runs at the laser repetition rate $(1 \mathrm{kHz})$; however, the rate at which coincidences occur is significantly below that $(<100 \mathrm{~Hz})$. An estimate of the microchannel plate detector efficiency (as a function of particle mass and kinetic energy) for heavy (>100 a.m.u.) ions can be found in (Fraser 2002). Using equation (26) in this reference, a kinetic energy of $1 \mathrm{keV}$ and an open area ratio of $55 \%$ for the MCP-s, we estimate the detection efficiencies of the cationic fragments, and use their ratio to correct the relative yields of the coincidence photoelectron spectra. For a fixed longitudinal accelerating voltage of 1 $\mathrm{kV}$, the values are spread between 5 and $50 \%$, depending on the mass of the fragment. For electrons, using $0.6 \mathrm{kV}$ for acceleration, the detection efficiency is about $50 \%$.

The steps for processing the data further is the same for both the regular and the coincidence VMI experiment. The 2D photoelectron momentum distributions are inverse-Abel transformed, and angularly integrated in the range of \pm 18 degrees about the laser polarization axis. This value was chosen because the angular extent of the features we wished to focus on in the $2 \mathrm{D}$ image is within this range. Finally, the angularly integrated yield is converted to a kinetic energy distribution.

While it is difficult to directly measure the resolution of our VMI apparatus given the lack of a tunable monoenergetic source of electrons, we can estimate a lower limit 
to the resolution of our VMI apparatus based upon the size of a single electron hit on the detector - the $1 / e^{2}$ diameter of such a hit is $\approx 2$ pixels. We argue that two such hits are just resolved if their center are separated by twice this width, i.e. 4 pixels. The mapping of electron velocity to camera pixel is linear $(\approx 5 \mathrm{~km} / \mathrm{s}$ per pixel), however, the mapping of energy is quadratic, giving nonuniform resolution across the spectrum. E.g., at $0 \mathrm{eV}, \Delta E \approx 1 \mathrm{meV}$; at $1 \mathrm{eV}, \Delta E \approx 70 \mathrm{meV}$, and at $2 \mathrm{eV}, \Delta E \approx 100 \mathrm{meV}$.

Figures 1 and 2 show the photoelectron spectra measured in coincidence with the two most prominent ionic fragments for two different molecules from the family of halomethanes. The most prominent fragments are $\mathrm{CH}_{2} \mathrm{Br}^{+}$in case of $\mathrm{CH}_{2} \mathrm{IBr}$ and $\mathrm{CH}_{2} \mathrm{Cl}^{+}$in case of $\mathrm{CH}_{2} \mathrm{BrCl}$. For strong field ionization of molecules with several low lying continua with comparable ionization potentials, one may expect significant structure in the low energy photoelectron spectrum. The kinetic energy of electrons ionized to the $i^{\text {th }}$ continuum is given by:

$$
K^{i}=n h \nu-I_{p}^{i}-U_{p}-E_{D S S}^{i}
$$

where $h \nu$ is the energy of a single photon (typically $1.6 \mathrm{eV}$ in these experiments - see discussion below), $I_{p}^{i}$ is the ionization potential associated with the $i^{\text {th }}$ continuum (or ionic state), $U_{p}$ is the ponderomotive potential and $E_{D S S}^{i}$ is the dynamic Stark-shift of the $i^{\text {th }}$ ionic state. Based on detailed calculations/measurements of each term in this expression, we can assign each of the peaks in the coincidence spectra to a specific continuum.

\section{Calculations}

Excitation energies of $\mathrm{CH}_{2} \mathrm{BrI}^{+}$at the Franck-Condon position taking into account spinorbit coupling between electronic states are taken from (Geißler et al. 2011). The equilibrium geometry of $\mathrm{CH}_{2} \mathrm{BrCl}$ in its ground electronic state is taken from (Rozgonyi \&González 2001). The excitation energies for the five lowest excited electronic states of $\mathrm{CH}_{2} \mathrm{BrCl}^{+}$were determined at the same level of theory as those for the other species (Geißler et al. 2011), i,e, using the multireference configuration interaction (MRCI) method implemented in the Molpro program package (Werner et al. 2012). The MRCI calculations were based on previous state-averaged complete active space self-consistent field (Roos et al. 1980) computations. The active space for both species consisted of 11 electrons on 8 active orbitals being the four non-bonding lone pairs of the halogen atoms and the $\mathrm{C}-\mathrm{Br}$ and $\mathrm{C}-\mathrm{Cl}$ bonding and antibonding sigma orbitals. The Douglas Kroll Hamiltonian (Douglas et al. 1974) was applied and the ANO-RCC basis sets (Widmark et al. 1990, Roos et al. 2004) were used with contractions 3s2p1d for H, 4s3p2d1f for C, 6s5p3d2f1g for $\mathrm{Br}$ and $5 \mathrm{~s} 4 \mathrm{p} 2 \mathrm{~d} 1 \mathrm{f}$ for $\mathrm{Cl}$ atoms. The spin-orbit coupling (SOC) among the ionic states were computed using atomic mean field integrals (Heß et al. 1996). The ionization potentials for the first five ionic states for both molecules can be found in table 1. In case of both molecules, values for the ground state $D_{0}$ were taken from experimental measurements by Lago et. al. (Lago et al. 2005). While 


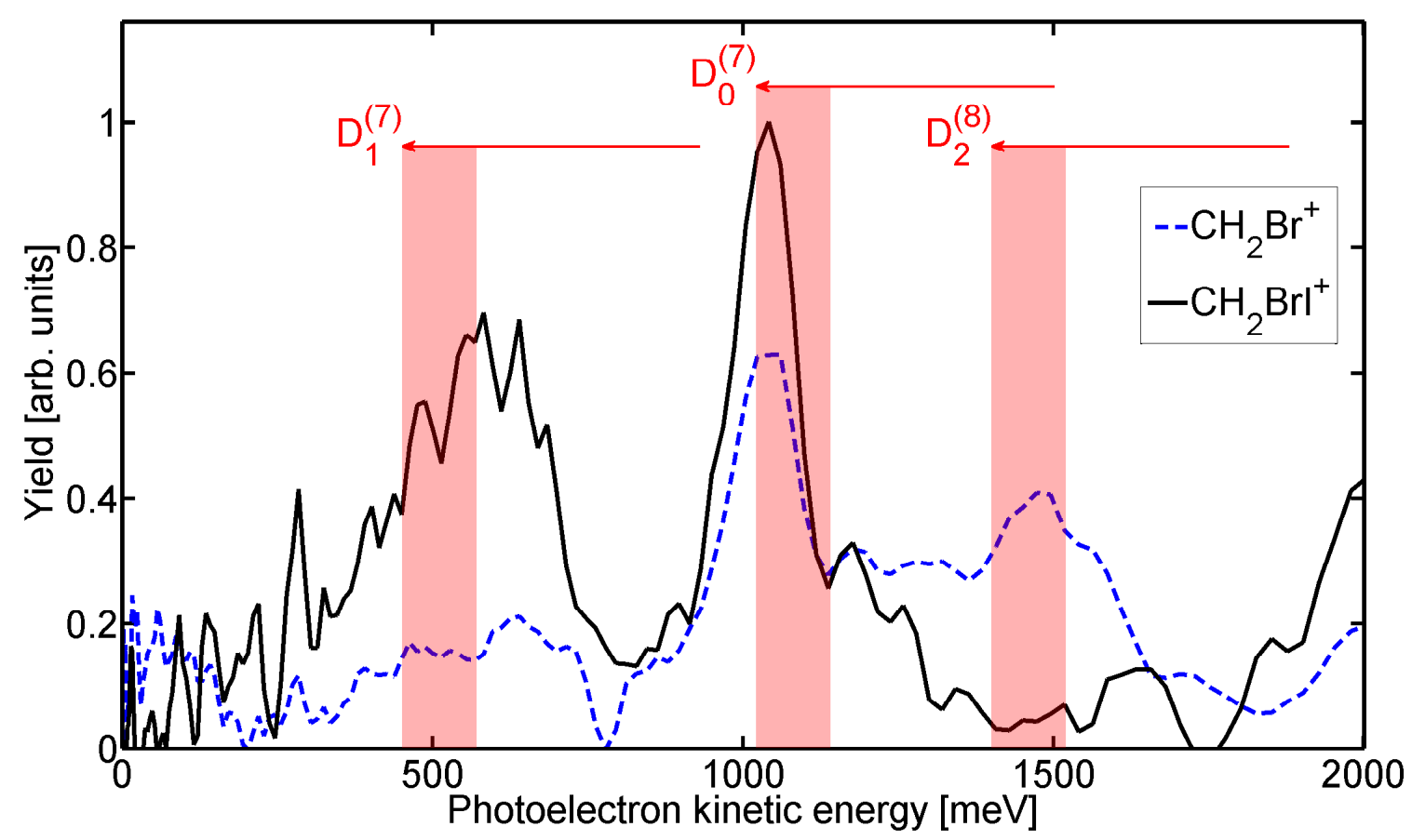

Figure 1: Photoelectron spectra for ionization of $\mathrm{CH}_{2} \mathrm{IBr}$. The dashed blue line shows the spectrum measured in coincidence with $\mathrm{CH}_{2} \mathrm{Br}^{+}$fragments, while the solid black line shows the spectrum measured in coincidence with the parent ion. Superscript on the state labels indicate the number of photons absorbed to access the specific continuum. Arrows indicate the range of peak locations allowed for the full range of ponderomotive shifts in the laser focus, whereas the shaded region highlights the expected peak locations given the arguments discussed in the text.

experimental values were not available for the excited ionic states, similar calculations for the molecule $\mathrm{CH}_{2} \mathrm{I}_{2}$ showed excellent agreement with experiment (less than $5 \%$ deviation) on the energies of the lowest four ionic states (Potts et al. 1970): the differences between calculated and measured excitation energies were less than $0.05 \mathrm{eV}$ in each case.

\begin{tabular}{c|c|c}
\hline & $\mathrm{CH}_{2} \mathrm{IBr}$ & $\mathrm{CH}_{2} \mathrm{BrCl}$ \\
\hline$D_{0}$ & 9.69 & 10.77 \\
$D_{1}$ & 10.26 & 11.03 \\
$D_{2}$ & 10.91 & 11.72 \\
$D_{3}$ & 11.12 & 11.81 \\
$D_{4}$ & 13.62 & 14.70 \\
\hline
\end{tabular}

Table 1: Ionization potentials in $\mathrm{eV}$. Energies for $\mathrm{D}_{0}$ are experimental values from Lago et. al., while higher lying states are calculated relative to $\mathrm{D}_{0}$ using the MRCI method as described in the text. 


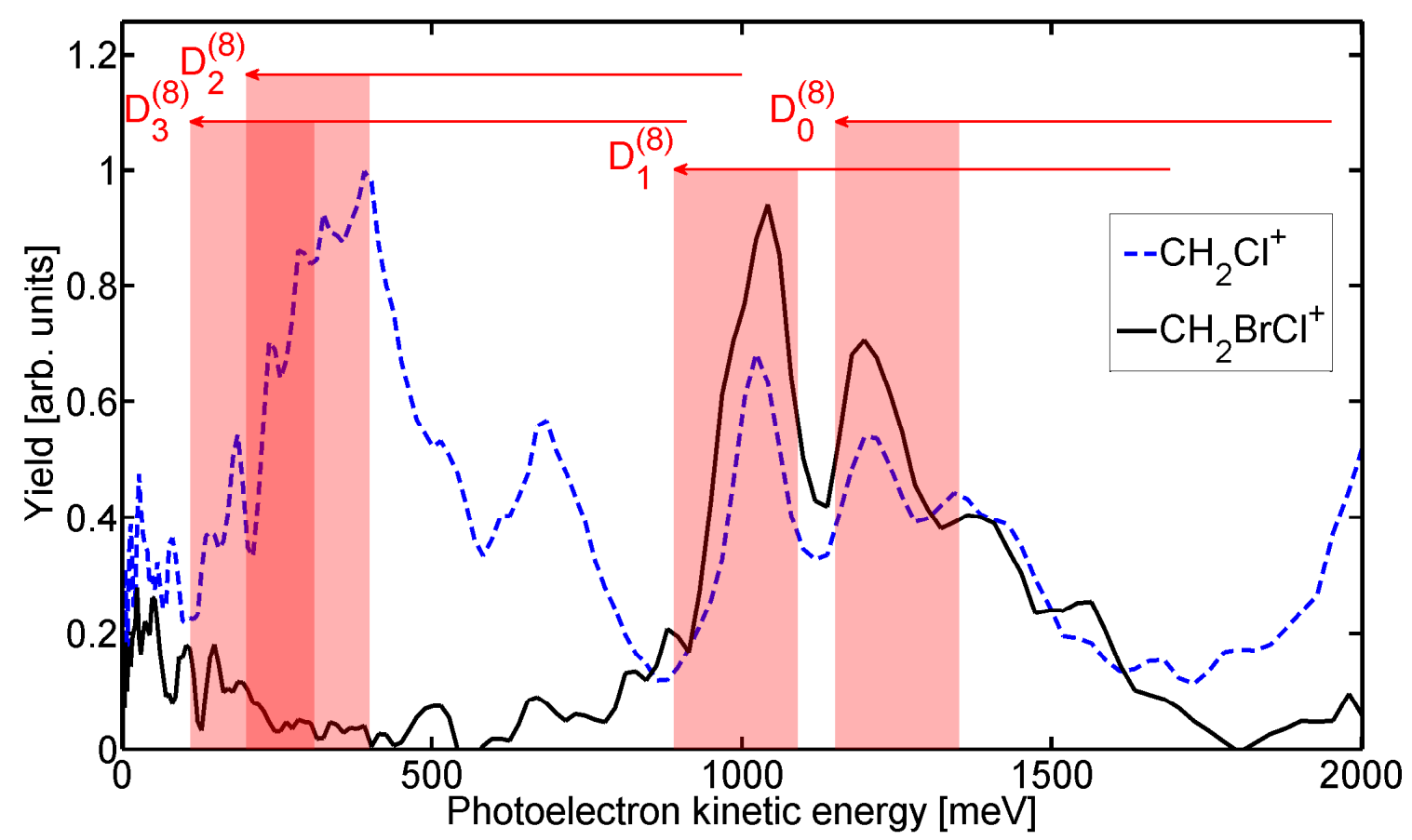

Figure 2: Photoelectron spectra for ionization of $\mathrm{CH}_{2} \mathrm{BrCl}$. The dashed blue line shows the spectrum measured in coincidence with $\mathrm{CH}_{2} \mathrm{Cl}^{+}$fragments, while the solid black line shows the spectrum measured in coincidence with the parent ion. Superscript on the state labels indicate the number of photons absorbed to access the specific continuum. Arrows indicate the range of peak locations allowed for the full range of ponderomotive shifts in the laser focus, whereas the shaded region highlights the expected peak locations given the arguments discussed in the text.

\section{Results}

The ponderomotive potential, $U_{p}$, describes the time-averaged kinetic energy of a charged particle in an electromagnetic field. It depends on the laser frequency, $\omega$, and intensity, I, but not on any molecular parameters $\left(U_{p}=\frac{2 e^{2} I}{4 \epsilon_{0} c m_{e} \omega^{2}}\right)$. In the shortpulse limit, the ionization potential for each electronic state is effectively increased by $U_{p}$ (Freeman et al. 1987), and this has to be taken into account when making the peak assignments. We note that since not all ionization takes place at the peak intensity, there will be a distribution of ponderomotive shifts for molecules ionized at intensities around the peak intensity. However, since the ionization is a nonlinear function of intensity, most of the ionization takes place near the peak intensity ( $70 \%$ of the ionization yield occurs for intensities within $25 \%$ of the peak intensity), and therefore we focus on peak ponderomotive shifts in our discussion below.

We measured the peak ponderomotive shifts using the intensity dependent shifting of peaks in the photoelectron spectrum for $\mathrm{CS}_{2}$. We chose $\mathrm{CS}_{2}$ because the photoelectron spectrum is much simpler than for $\mathrm{CH}_{2} \mathrm{IBr}$ or $\mathrm{CH}_{2} \mathrm{BrCl}$ and because we measured a linear shift in the peak locations with intensity (ponderomotive shifting), indicating that for the range of intensities used in the calibration, intermediate resonances do not play an important role in determining the peak locations. The energy difference between the 


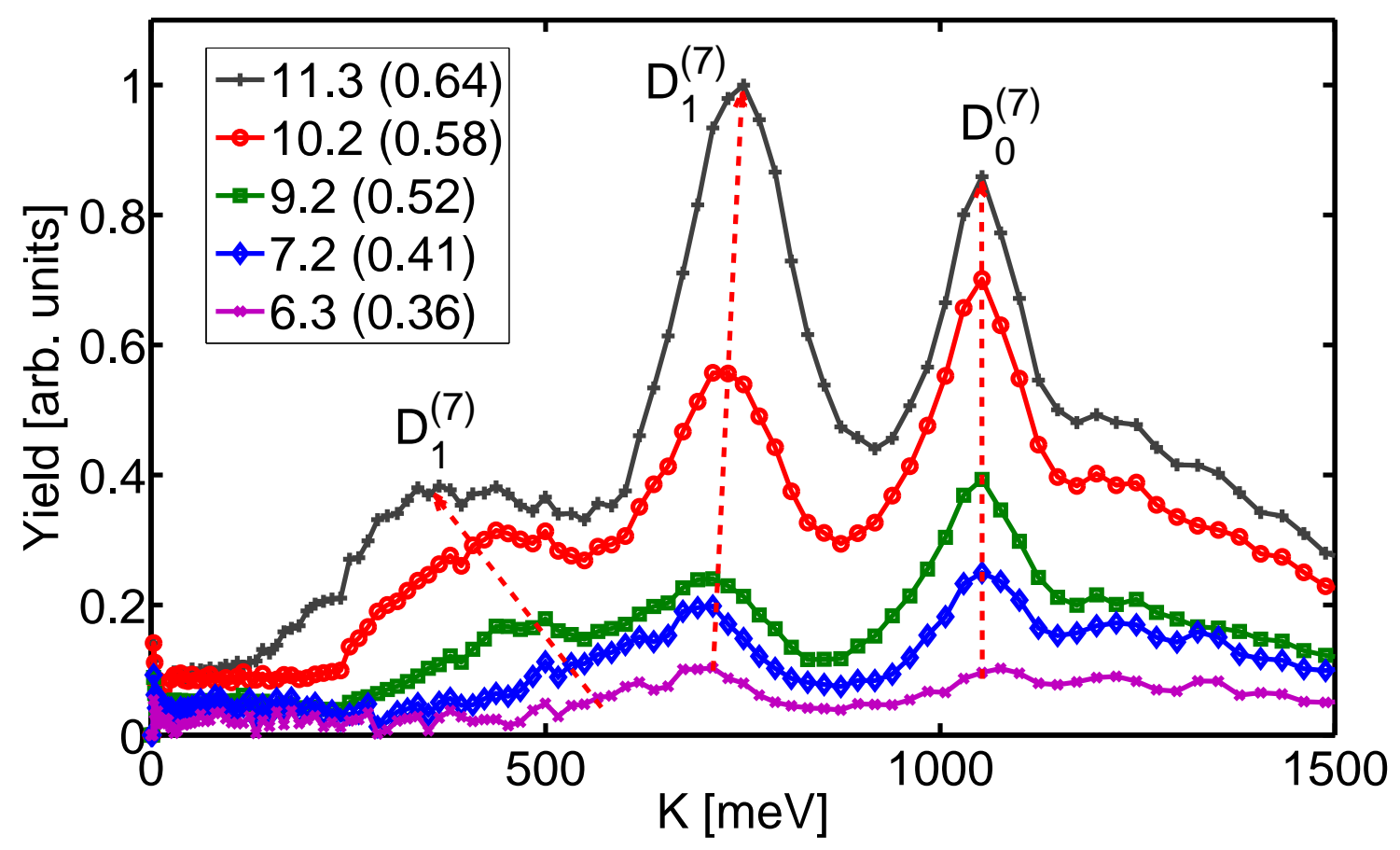

Figure 3: Photoelectron spectrum for $\mathrm{CH}_{2} \mathrm{IBr}$ for several different laser intensities. Superscript on the state labels indicate the number of photons absorbed to access the specific continuum. Legend: laser peak intensity in $\mathrm{TW} / \mathrm{cm}^{2}$ (and ponderomotive potential in $\mathrm{eV}$ ).

ground and first excited state of the cation is $2.6 \mathrm{eV}$ (Potts \& Fattahallah 1980), which means that for a large range of intensities it is possible to ionize only to the ground state.

Figures 1 and 2 show the photoelectron spectra for $\mathrm{CH}_{2} \mathrm{IBr}$ and $\mathrm{CH}_{2} \mathrm{BrCl}$, respectively. In each figure, photoelectron spectra measured in coincidence with the parent ion (black solid line) and with the most abundant fragment (blue dashed line) can be found. Horizontal arrows indicate regions where photoelectrons coming from the lowest-lying electronic states of the ion contribute to the spectrum; the tail of each arrow indicate the appearance energy of the photoelectrons at zero field (zero ponderomotive potential), while the head of each arrow shows the same for the peak of the field (peak ponderomotive potential). The red shaded areas further highlight regions of the spectrum where the $U_{p}$ is within $25 \%$ of its peak value for each state, since as noted above, most of the ionization takes place within this range of the ponderomotive shift.

\section{Discussion}

In assigning the peaks in the photoelectron spectrum of $\mathrm{CH}_{2} \mathrm{IBr}$ to specific ionic states, we first note that $\mathrm{D}_{4}$ has a significantly higher ionization potential than states $\mathrm{D}_{0}$ to $\mathrm{D}_{3}$. This suggests that ionization to $\mathrm{D}_{4}$ should be suppressed with respect to the lower states. 


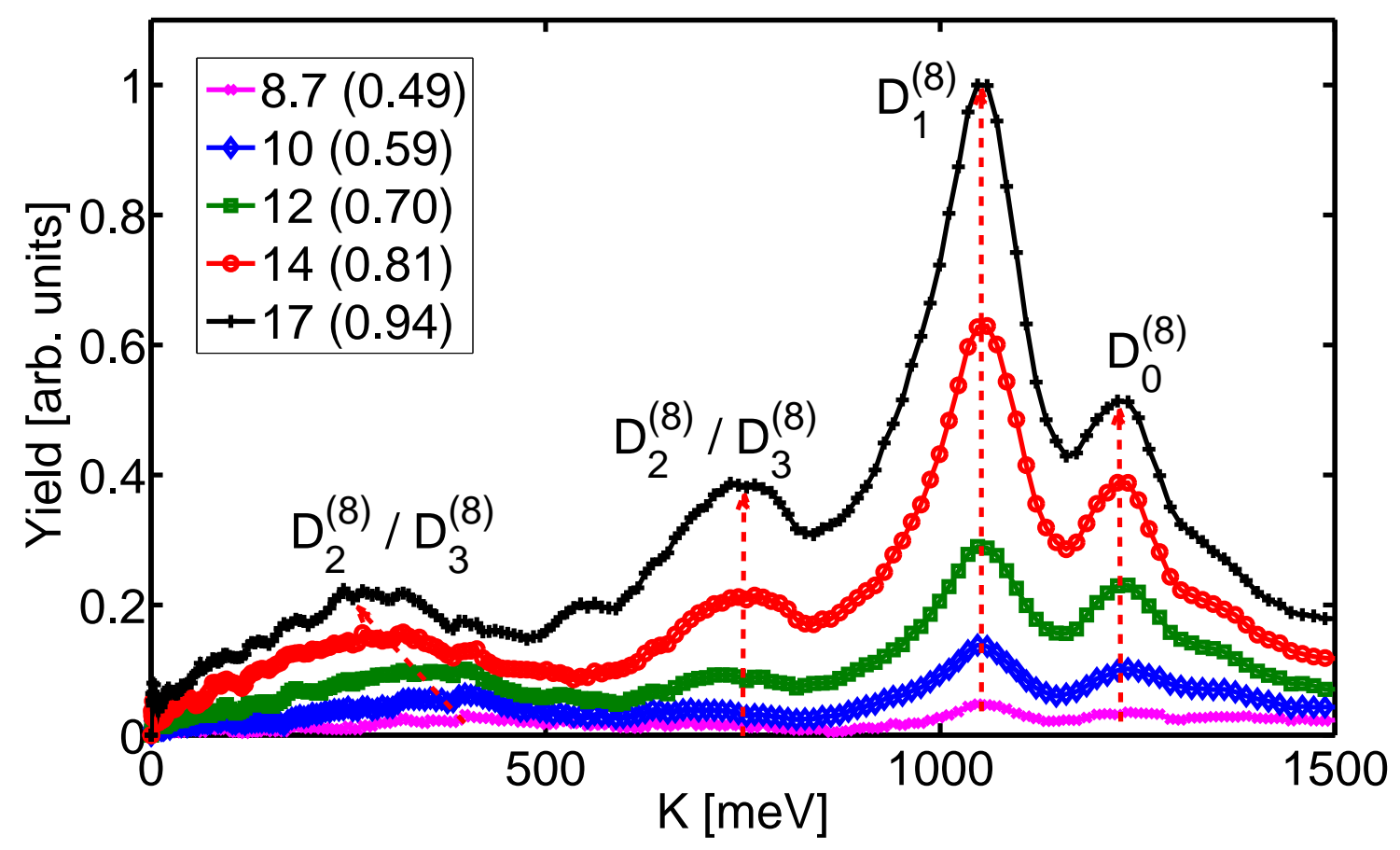

Figure 4: Photoelectron spectrum for $\mathrm{CH}_{2} \mathrm{BrCl}$ for several different laser intensities. Superscript on the state labels indicate the number of photons absorbed to access the specific continuum. Legend: laser peak intensity in $\mathrm{TW} / \mathrm{cm}^{2}$ (and ponderomotive potential in $\mathrm{eV}$ ).

Our measurements of the ion TOFMS are consistent with this expectation in that we measure very few fragment ions coming from ionization to $\mathrm{D}_{4}$, which can fragment to form $\mathrm{I}^{+}$and $\mathrm{CH}_{2} \mathrm{I}^{+}$(Lago et al. 2005). Furthermore, earlier measurements performed with velocity map imaging of the ionic fragments found that $\mathrm{CH}_{2} \mathrm{Br}^{+}$produced with kinetic energy less than $0.30 \mathrm{eV}$ could be associated with dissociation on $\mathrm{D}_{2}$ or $\mathrm{D}_{3}$, whereas $\mathrm{CH}_{2} \mathrm{Br}^{+}$produced with a kinetic energy above $0.50 \mathrm{eV}$ could be associated with $\mathrm{D}_{4}$. Measurements carried out at the same intensity as the measurements shown in this paper did not find any $\mathrm{CH}_{2} \mathrm{Br}^{+}$with kinetic energy above $0.50 \mathrm{eV}$, consistent with the idea that there is no ionization to $\mathrm{D}_{4}$ in the present measurements (Geißler et al. 2011). Thus, we restrict our interpretation of the spectrum to ionization to $\mathrm{D}_{0}$ through $\mathrm{D}_{3}$. We also note that for both $\mathrm{CH}_{2} \mathrm{IBr}$ and $\mathrm{CH}_{2} \mathrm{BrCl}$, the lowest two ionic states, $\mathrm{D}_{0}$ and $\mathrm{D}_{1}$, are bound, while $\mathrm{D}_{2}$ and $\mathrm{D}_{3}$ are dissociative (Geißler et al. 2011, Lago et al. 2005). Finally we note that the photoelectron energy is determined at the moment of ionization, and thus transitions in the ion driven by the laser do not affect the photoelectron spectrum. Thus, we argue that peaks measured in coincidence with the parent ion must come from ionization to $\mathrm{D}_{0}$ and $\mathrm{D}_{1}$. Peaks measured in coincidence with the fragment ion are more subtle, but comparison with the spectrum measured in coincidence with the parent can distinguish between different cases. Peaks in the spectrum measured in coincidence with the fragment, but which do not appear in the spectrum measured in coincidence with the parent, can be associated with direct ionization to dissociative states $\mathrm{D}_{2}$ and $\mathrm{D}_{3}$, 
whereas peaks that appear in both spectra can be associated with indirect ionization to $\mathrm{D}_{2}$ or $\mathrm{D}_{3}$ (i.e. ionization to $\mathrm{D}_{0}$ or $\mathrm{D}_{1}$ followed by laser driven transitions in the ion).

Given these considerations, it is natural to assign the peaks in the $\mathrm{CH}_{2} \mathrm{BrI}$ spectrum at $1.04 \mathrm{eV}$ and $\approx 0.55 \mathrm{eV}$ to $\mathrm{D}_{0}$ and $\mathrm{D}_{1}$ and the peak at $1.45 \mathrm{eV}$ to $\mathrm{D}_{2}$ or $\mathrm{D}_{3}$. We note that peak at $0.55 \mathrm{eV}$ shifts with intensity (see figure 3), while the peaks at 1.04 and $1.45 \mathrm{eV}$ do not. This is because the peaks at 1.04 and $1.45 \mathrm{eV}$ are due to resonantly enhanced ionzation (via Freeman resonances (Freeman et al. 1987, Gibson et al. 1992)), whereas the peak at $0.55 \mathrm{eV}$ is not resonantly enhanced. In order to test this preliminary assignment of the peaks, we consider the energies we expect for these peaks based on the formula given above, assuming that each peak is generated near the peak intensity of the pulse and therefore experiences the peak ponderomotive shift. For this case of maximal ponderomotive shift, and considering the lowest order process that would lead to a positive photoelectron energy, we expect the peak corresponding to $\mathrm{D}_{0}$ to be at $K=7 \cdot 1.60-9.69-0.48=1.03 \mathrm{eV}$, which compares favorably with the measured 1.04 $\mathrm{eV}$. For $\mathrm{D}_{2}, 7$ photon ionization is not energetically allowed, and therefore we expect $K=8 \cdot 1.60-10.91-0.48=1.41 \mathrm{eV}$, which again agrees well with the experiment. For the peak assigned to $\mathrm{D}_{1}$, the predicted energy is $K=7 \cdot 1.60-10.26-0.48=0.46 \mathrm{eV}$. This again is in reasonable agreement with the experimental measurements, confirming our initial assignments. It is natural to look for evidence of ionization to $\mathrm{D}_{3}$, given the small difference in ionization potential between $\mathrm{D}_{2}$ and $\mathrm{D}_{3}$. The expected location for a peak corresponding to $\mathrm{D}_{3}$ is $K=8 \cdot 1.60-11.12-0.48=1.20 \mathrm{eV}$, at which we do see a nonzero yield, although there is not a well defined peak. Therefore it is difficult to draw a firm conclusion as to whether or not there is substantial ionization to $\mathrm{D}_{3}$.

Similar arguments can be made to assign the peaks in the spectrum for $\mathrm{CH}_{2} \mathrm{BrCl}$, taking into account that for these measurements the laser was tuned slightly to the red and thus the photon energy was $1.59 \mathrm{eV}$. Also, given the higher ionization potential of this molecule, we worked at higher intensities to get a comparable yield and thus the peak ponderomotive shift is $0.80 \mathrm{eV}$. Again, we focus our attention to the four lowest-lying states, since as in the case of $\mathrm{CH}_{2} \mathrm{IBr}, \mathrm{D}_{4}$ is much higher in energy than the states below it. We point out that the two lowest-lying ionic states, $\mathrm{D}_{0}$ and $\mathrm{D}_{1}$ are not dissociative, while $\mathrm{D}_{2}$ and $\mathrm{D}_{3}$ are, leading mostly to the production of $\mathrm{CH}_{2} \mathrm{Cl}^{+}$ (Lago et al. 2005). Peaks corresponding to the first two of the four states mentioned are expected to be found in the spectra associated with the parent ion, as a result of direct ionization. However, we expect that some of these may also be found in the spectra of the fragment, which can be explained by post-ionization transitions of the ion from a non-dissociative to a dissociative state. Additionally, it is clear that in the spectrum of the parent, no peaks are expected to be seen corresponding to dissociative states.

In the spectrum associated with the parent ion, two narrow peaks are visible at 1.04 and $1.20 \mathrm{eV}$, which also show up in the spectrum of the fragment (see figure 2). These, based on the above arguments can be assigned to the states $D_{1}$ and $\mathrm{D}_{0}$, respectively. The expected kinetic energy for electrons associated with $\mathrm{D}_{0}$ is 
$K=8 \cdot 1.59-10.77-0.80=1.15 \mathrm{eV}$ and for $\mathrm{D}_{1}$ it is $K=8 \cdot 1.59-11.03-0.80=0.89 \mathrm{eV}$ at the maximum ponderomotive shift. The observed appearance energies lie well within the range set between the $75 \%$ and the peak ponderomotive shift, which supports the assignment.

The spectrum associated with the fragment ion (figure 2) shows two features that are absent from the parent spectrum: a broad feature between 0.10 and $0.50 \mathrm{eV}$ (centered at $0.30 \mathrm{eV}$ ), and a relatively narrow one centered at $0.70 \mathrm{eV}$. The peak at $0.30 \mathrm{eV}$ can be associated with both $\mathrm{D}_{2}$ and $\mathrm{D}_{3}$ since the expected energies for the two states are close together and both fall within this broad peak. The expected energies for $\mathrm{D}_{2}$ and $\mathrm{D}_{3}$ are $K=8 \cdot 1.59-11.72-0.80=0.20 \mathrm{eV}$ and $K=8 \cdot 1.59-11.81-0.80=0.11$ $\mathrm{eV}$ respectively. However, the origin of the $0.70 \mathrm{eV}$ peak is unclear. Looking at the intensity dependent measurements shown in figure 4, one can see that this peak and the peak at $\approx 0.30 \mathrm{eV}$ in the coincidence spectrum have a common energy for a low intensity which corresponds to an intensity close to where the peak(s) appears in the spectrum. This, in conjunction with the fact that the peak is in coincidence with the fragment ion suggest that it is associated with ionization to either $\mathrm{D}_{2}$ or $\mathrm{D}_{3}$. As it does not shift with intensity, it is resonantly enhanced, and the position in the spectrum corresponds to the resonant enhancement occurring at about $1 / 3$ of the peak ponderomotive shift.

In addition to the radial distributions discussed above, the velocity map imaging measurements of the photoelectrons also provide angular distributions. While the angledependent yields are not the focus of the current analysis, we note that the angular distributions for the $0.30 \mathrm{eV}$ and $0.70 \mathrm{eV}$ peaks are the same (within the statistical uncertainty of our measurements), but different from the $1.04 \mathrm{eV}$ and $1.20 \mathrm{eV}$ peaks. This is consistent with the idea that the 0.30 and $0.70 \mathrm{eV}$ peaks are both due to ionization to $\mathrm{D}_{2} / \mathrm{D}_{3}$.

As noted above, resonances in both the neutral and the ion play an important role in the ionization dynamics. The intensity dependence of the photoelectron spectrum highlights the role of neutral resonances, and the comparison of photoelectron spectra in coincidence with the parent and fragment ions highlights the role of ionic resonances. In order to illustrate the role of ionic resonances and to distinguish between direct and indirect ionization to a given final ionic state, we turn to figure 5. This figure shows how the direct and the indirect ionization pathways can be associated with different peaks in the coincidence photoelectron spectra. We would like to point out that the appearance of a peak corresponding to the nondissociative state $\mathrm{D}_{0}$ in the fragment spectrum signifies that accessing a dissociative state (most likely $\mathrm{D}_{2}$ or $\mathrm{D}_{3}$ ) took place in at least two steps (first ionization, then a transition in the ion). Hence we call this pathway 'indirect' - this is to be contrasted with the peak corresponding to $\mathrm{D}_{2}$, which is associated with a 'direct' multiphoton transition from $\mathrm{S}_{0}$ (the neutral ground state) to $\mathrm{D}_{2}$.

As noted briefly above, the intensity dependence of the photoelectron spectra (shown in figures 3 and (4) allows us to distinguish between resonance enhanced ion- 


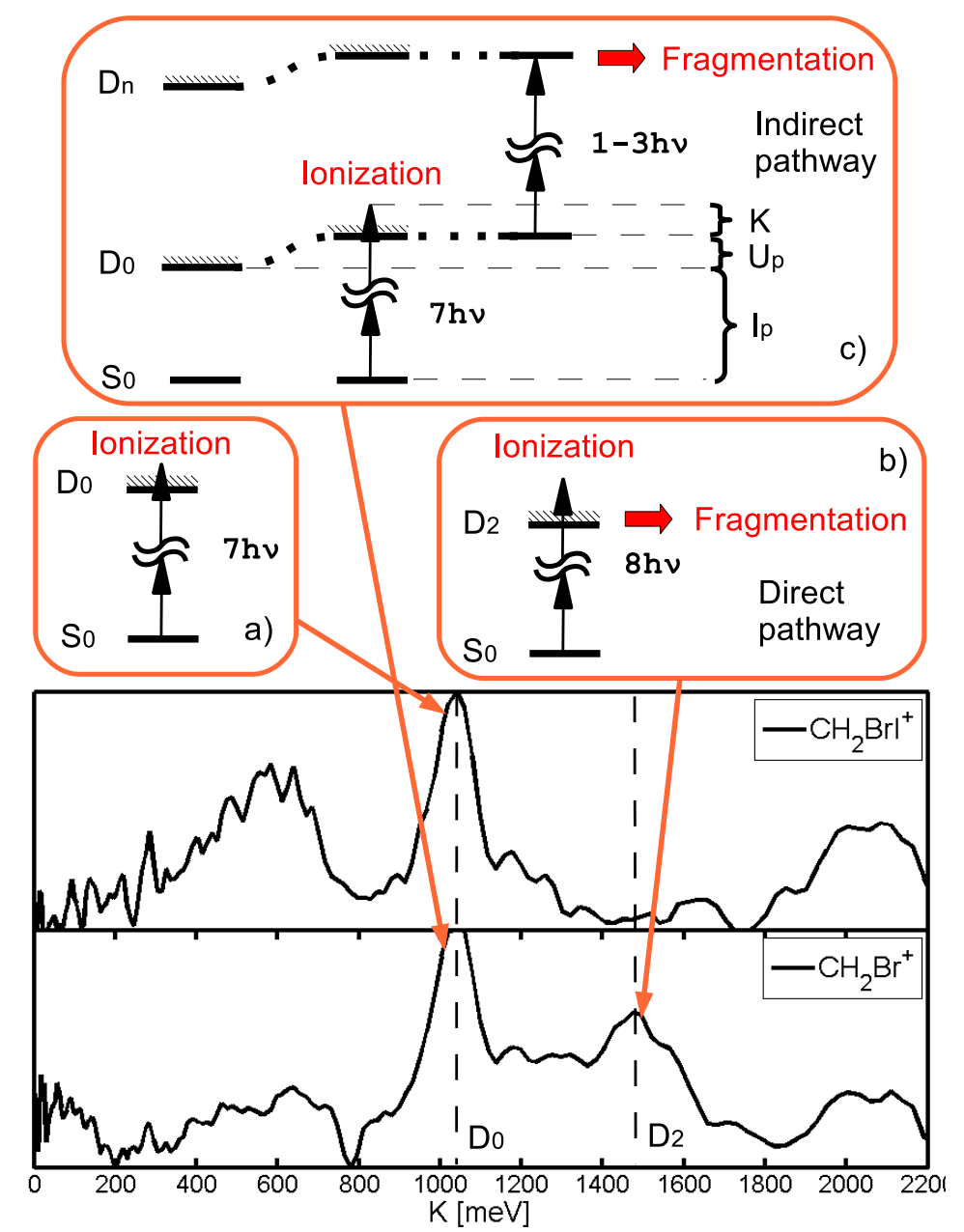

Figure 5: Illustration of dissociative and non-dissociative pathways in the ion, and their signature in the measured photoelectron spectra associated with the parent and fragment cations of $\mathrm{CH}_{2} \mathrm{IBr}$. a) direct 7-photon ionization to the nondissociative continuum $\mathrm{D}_{0}$. b) direct 8-photon ionization to the dissociatiove continuum $\mathrm{D}_{2}$. c) 7-photon ionization to the non-dissociative continuum $\mathrm{D}_{0}$, followed by a post-ionization transition to a higher-lying dissociative state $\left(\mathrm{D}_{n}\right)$. Arrows point to the corresponding peaks in the spectra.

ization and non-resonant ionization. These measurements are not made in coincidence mode. The peaks which don't shift ponderomotively with intensity correspond to resonantly enhanced ionization. These are the $1.04 \mathrm{eV}$ peak for $\mathrm{D}_{0}$ and the $0.70 \mathrm{eV}$ peak for $\mathrm{D}_{1}$ in $\mathrm{CH}_{2} \mathrm{IBr}$; also the $1.20 \mathrm{eV}$ peak for $\mathrm{D}_{0}$, the $1.04 \mathrm{eV}$ peak for $\mathrm{D}_{1}$ and the 0.75 $\mathrm{eV}$ peak for $\mathrm{D}_{2} / \mathrm{D}_{3}$ in $\mathrm{CH}_{2} \mathrm{BrCl}$. However, we also point out broad features that shift to lower appearance energies as the laser peak intensity increases. One shifts from 0.50 to $0.30 \mathrm{eV}$ in $\mathrm{CH}_{2} \mathrm{IBr}$ and we assign this to a nonresonant contribution from $\mathrm{D}_{1}$. The other is observed in $\mathrm{CH}_{2} \mathrm{BrCl}$ and shifts from 0.40 to $0.25 \mathrm{eV}$, and this we attribute to $\mathrm{D}_{2} / \mathrm{D}_{3}$ as was previously discussed. Based on the intensity dependent spectra, it is clear that strong field ionization in these two molecules proceeds via a mixture of resonance 
enhanced and non-resonant ionization.

\section{Conclusion}

In conclusion, we have analyzed the velocity map imaged spectrum of photoelectrons resulting from strong field ionization in coincidence with fragment ions. Our measurements, in conjunction with electronic structure and dynamic Stark shift calculations reveal the production of multiple ionic states via strong field ionization, and show evidence for post-ionization transitions in the ion.

The authors gratefully acknowledge support from the National Science Foundation under award number 1205397 and the Hungarian National Development Agency under grant number KTIA_AIK_12-1-2012-0014.

\section{Appendix I - True vs false coincidence rates}

We can estimate the ratio of true and false coincidences if we assume that the number of generated electron-ion pairs (assuming one ionic fragment per electron) per laser shot exhibits a Poisson distribution with the expectation value $\lambda$ and the number of occurrences $k$ :

$$
P(k, \lambda)=\frac{\lambda^{k} e^{-\lambda}}{k !}
$$

If we denote electron and ion detection efficiencies by $\eta_{e}$ and $\eta_{i}$, respectively, then the true coincidence probability, $T(\lambda)$, i.e., detecting exactly one electron with its corresponding ion, is

$$
T(\lambda)=\sum_{k \geqq 1} k \eta_{e} \eta_{i}\left(1-\eta_{e}\right)^{k-1}\left(1-\eta_{i}\right)^{k-1} \frac{\lambda^{k} e^{-\lambda}}{k !}
$$

The false coincidence probability, $F(\lambda)$, i.e., the detected electron-ion pair does not come from the same molecule is:

$$
F(\lambda)=\sum_{k \geqq 2} k(k-1) \eta_{e} \eta_{i}\left(1-\eta_{e}\right)^{k-1}\left(1-\eta_{i}\right)^{k-1} \frac{\lambda^{k} e^{-\lambda}}{k !}
$$

A desired true-to-false coincidence ratio,

$$
R(\lambda)=\frac{T(\lambda)}{F(\lambda)}=\frac{1}{\lambda\left(1-\eta_{e}\right)\left(1-\eta_{i}\right)}
$$

dictates the expectation value, $\lambda$, and consequently, the pulse intensity and molecular beam density that are used. In our case, we estimate $\eta_{e}=0.5, \eta_{i}=0.25$ and aim for $R(\lambda) \geq 5$, then we have $\lambda \approx 0.5$. In other words, by adjusting the molecular beam density and pulse intensity to obtain $\lambda \approx 0.5,83 \%$ of our data is true coincidence. 


\section{Appendix II - Dynamic Stark shift calculations}

In order to estimate the dynamic Stark shift of each ionic state, $\mathrm{E}_{D S S}^{i}$, we performed a numerical integration of the time-dependent Schrödinger equation including the molecular ground state and the five lowest ionic states of the $\mathrm{CH}_{2} \mathrm{BrCl}$ molecule. This calculation was motivated by the fact that given the various detunings between the low lying ionic states and the laser, one cannot make the rotating wave approximation or perform adiabatic elimination. The rotating wave approximation requires the detunings to be much smaller than the laser frequency and adiabatic elimination requires that the detunings be larger compared to the Rabi frequency. Neither approximation is valid for all states, leading to rapidly oscillating terms in the Hamiltonian which one would diagonalize in order to calculate dressed state energies and Stark shifts. In order to average over these rapidly oscillating terms, as is relevant for our experimental measurements, we 'probed' the ionic state energies by looking for population transfer from the ground state as a function of UV laser frequency. The coupling between the neutral ground state and the ionic states is artificial, but allows us to probe the energy shift of the ionic states as a function of ionization pulse intensity. Only five ionic states are considered because there is a substantial energy gap to the next lowest ionic states. To model the real laser field used in the experiment, which are responsible for both the Stark shift and multiphoton ionization, we defined two fields in the calculation: a strong IR laser field inducing the Stark shift and a weak VUV field ionizing the ground state. By calculating the ionic states' population as a function of IR field strength and VUV photon energy, we can map out the Stark shift of each ionic state as a function of IR field strength.

The transition dipole moments (TDMs) between ionic states, state energies and spin-orbit couplings for the ions are based on the $a b$ initio electronic structure calculations described in the calculation section, while the TDMs between the neutral and ionic states were set to 0.1 a.u. The total electronic Hamiltonian (nuclear dynamics is not considered here) consists of 3 parts, the bare Hamiltonian $H_{0}$ (not including spin-orbit coupling), spin-orbit coupling $H_{S O}$, and the molecule-field dipole-coupling $H_{M F}$ :

$$
\begin{aligned}
& H=H_{0}+H_{S O}+H_{M F} \\
& H_{M F}=-\vec{\mu} \cdot \vec{E} \\
& \left(H_{0}+H_{S O}\right)\left|\phi_{i}\right\rangle=\hbar \omega_{i}\left|\phi_{i}\right\rangle
\end{aligned}
$$

The calculation is carried out in the eigenspace of $H_{0}+H_{S O}$ (i.e. in the spin-orbit adiabatic basis). The wave function is written in terms of the eigenstates:

$$
|\psi(t)\rangle=\tilde{a_{0}}(t)\left|\phi_{0}\right\rangle+\sum_{i \neq 0} \tilde{a_{i}}(t)\left|\phi_{i}\right\rangle
$$




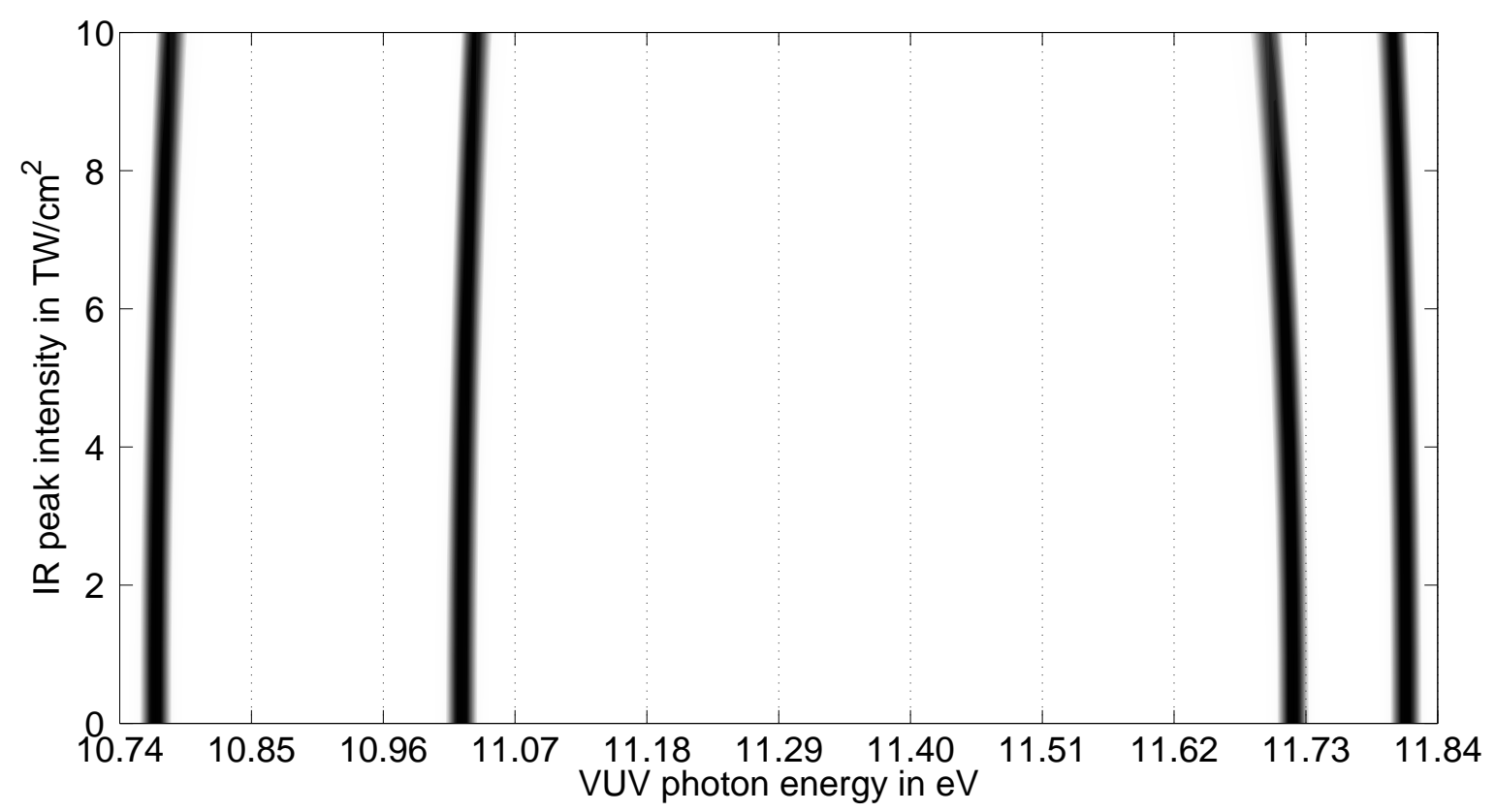

Figure 6: Calculations aimed at estimating dynamic Stark shifts for ionic states of $\mathrm{CH}_{2} \mathrm{BrCl}$ in a strong field IR laser pulse. The graph shows population of the first five ionic states as a function of IR field strength and VUV photon energy. On the horizontal axis the frequency of the VUV radiation is plotted, while on the vertical, it is the peak intensity of the IR pulse.

Substituting (6) and (9) into the Schrödinger equation $i \hbar \frac{\partial}{\partial t}|\psi\rangle=H|\psi\rangle$ and transforming to the rotating frame $\tilde{a}_{i}(t)=a_{i}(t) e^{-i \omega_{i} t}$, we arrive at :

$$
\begin{aligned}
& \dot{a}_{0}(t)= \frac{i}{\hbar} \sum_{i \neq 0} \mu_{0 i}\left[\mathcal{E}_{V U V}(t) e^{i \omega_{V U V} t}+\mathcal{E}_{I R}(t)\left(e^{-i \omega_{I R} t}+e^{i \omega_{I R} t}\right)\right] a_{i}(t) e^{-i \omega_{i 0} t} \\
& \dot{a}_{i \neq 0}= \frac{i}{\hbar} \mu_{i 0}\left[\mathcal{E}_{V U V}(t) e^{-i \omega_{V U V} t}+\mathcal{E}_{I R}(t)\left(e^{-i \omega_{I R} t}+e^{i \omega_{I R} t}\right)\right] a_{0}(t) e^{-i \omega_{0 i} t} \\
&+\frac{i}{\hbar} \sum_{j \neq i} \mu_{i j} \mathcal{E}_{I R}(t)\left(e^{-i \omega_{I R} t}+e^{i \omega_{I R} t}\right) a_{j}(t) e^{-i \omega_{j i} t} \\
& \omega_{i j}=\omega_{i}-\omega_{j} \quad i, j=1 . .10 \\
& \mathcal{E}_{V U V}(t)=E_{V U V} e^{-\frac{t^{2}}{2 \tau^{2}}} \quad \mathcal{E}_{I R}(t)=E_{I R} e^{-\frac{t^{2}}{2 \tau^{2}}}
\end{aligned}
$$

We have omitted the rapidly rotating terms, $\mathcal{E}(t) e^{ \pm i\left(\omega_{V U V}+\omega_{i o}\right) t}$, for $\omega_{V U V} \approx \omega_{i o}$, invoking the rotating wave approximation only for the VUV field, but not for the IR. Consequently, no simple analytical solution can be obtained. In figure 6 we show the result of a calculation for $\mathrm{CH}_{2} \mathrm{BrCl}$. The two-dimensional plot shows the total ionic population for a range of VUV photon energies (horizontal axis) and IR peak intensities (vertical axis). The calculation shows that population can be transferred to the lowest four ionic states when the VUV pulse is resonant with the energy difference between the neutral ground state and each of the ionic states, with energies given in table 1 . As the IR field strength increases, it couples the ionic states, leading to dynamic Stark 
shifts, as seen in shifts of the absorption peaks on the graph. We note that simulations were performed with the IR electric field polarized along the $\mathrm{C}$-Br bond. It is along this direction that the molecule-field coupling is the largest. Nevertheless, the shifts of the absorption peaks stay below $100 \mathrm{meV}$ for the intensities used in the measurements (see figure 6). Similar calculations for $\mathrm{CH}_{2} \mathrm{IBr}$ show similar Stark shifts. Since the other terms in equation 1 are significantly larger, we argue that neglecting $E_{D S S}$ is a good approximation.

Agostini P \& DiMauro L 2004 Reports on Progress in Physics 67, 813.

Boguslavskiy A E, Mikosch J, Gijsbertsen A, Spanner M, Patchkovskii S, Gador N, Vrakking M J J \& Stolow A 2012 Science 335(6074), 1336-1340.

Douglas M \&Kroll N.M 1974 Annals of Physics 81, pp. 89-155

Eppink A T J B \& Parker D H 1997 Review of Scientific Instruments 68(9), 3477-3484.

Fraser G 2002 International Journal of Mass Spectrometry 215(13), 13 - 30. Detectors and the Measurement of Mass Spectra.

Freeman R R, Bucksbaum P H, Milchberg H, Darack S, Schumacher D \& Geusic M E 1987 Phys. Rev. Lett. 59, 1092-1095.

Geißler D, Rozgonyi T, González-Vázquez J, González L, Marquetand P \& Weinacht T C 2011 Phys. Rev. A 84, 053422.

Gibson G N, Freeman R R \& McIlrath T J 1991 Physical Review Letters 67(10), 1230-1233.

Gibson G N, Freeman R R \& McIlrath T J 1992 Physical Review Letters 69, 1904-1907.

Heß B.A, Marian C.M, Wahlgren U \&Gropen O 1996 Chem. Phys. Lett. 251, pp. 365-371

Kling M \& Vrakking M 2008 Annu. Rev. Phys. Chem. 59, 463-492.

Kotur M, Zhou C, Matsika S, Patchkovskii S, Spanner M \& Weinacht T C 2012 Phys. Rev. Lett. 109, 203007.

Krausz F \& Ivanov M 2009 Rev. Mod. Phys. 81, 163-234.

Lago A F, Kercher J P, Bödi A, Sztáray B, Miller B, Wurzelmann D \& Baer T 2005 The Journal of Physical Chemistry A 109(9), 1802-1809.

Lehmann C S, Ram N B \& Janssen M H M 2012 Review of Scientific Instruments 83(9).

Li W, Jaroń-Becker A, Hogle C, Sharma V, Zhou X, Becker A, Kapteyn H \& Murnane M 2010 Proceedings of the National Academy of Sciences 107(47), 20219.

Li W, Zhou X, Lock R, Patchkovskii S, Stolow A, Kapteyn H C \& Murnane M M 2008 Science 322(5905), 1207-1211.

McFarland B K, Farrell J P, Bucksbaum P H \& Gühr M 2008 Science 322(5905), 1232-1235.

Potts A W \& Fattahallah G H 1980 Journal of Physics B: Atomic and Molecular Physics 13(13), 2545.

Potts A W, Lempka H J, Streets D G \& Price W C 1970 Philosophical Transactions of the Royal Society of London. Series A, Mathematical and Physical Sciences 268(1184), pp. 59-76.

Roos B.O, Taylor P.R \&Siegbahn P.E.M 1980 Chem. Phys. 48, pp. 157-173

Roos R.O, Lindh R, Malmquist P-A, Veryazov V \&Widmark P-O 2004 J. Phys. Chem. A 108, pp. $2851-2858$

Rozgonyi T \& González L 2001 Chem. Phys. Lett. 350, pp. 155-164.

Spanner M, Patchkovskii S, Zhou C, Matsika S, Kotur M \& Weinacht T C 2012 Phys. Rev. A 86, 053406.

H.-J. Werner, Knowles P.J, Knizia G., Manby F.R, Schütz M \&others 2012 MOLPRO, version 2012.1, a package of ab initio programs

Widmark P-O, Malmquist P-A, \&Roos B.O 1990 Theoretica Chimica Acta 77, pp. 291-306 